\title{
A flexible gel pad as an effective medium for scanning irregular surface anatomy
}

\author{
Ban C. H. Tsui, MD $\cdot$ Jenkin Tsui
}

Received: 7 October 2011/ Accepted: 26 October 2011/Published online: 4 November 2011

(C) Canadian Anesthesiologists' Society 2011

\section{To the Editor,}

Ultrasonography involves the use of high-frequency sound waves to produce a visual image of an internal body structure, and the method is rapidly gaining popularity as a tool to guide a variety of medical procedures. The primary obstacle when using ultrasound is attenuation of the ultrasound waves due to poor probe-surface contact, air pockets, and subtle changes in interface material. To overcome attenuation, liquid gel is normally used as a sound medium, while alternative mediums such as normal saline and dextrose $5 \%$ in water (D5W) have also been used to provide a smooth surface area. ${ }^{1,2}$ Nevertheless, a drawback of any liquid medium is the difficulty to apply and maintain a consistent contact surface for irregular anatomy surfaces, e.g., the anterior neck region when scanning the airway.

Recently, we described a simple and reusable phantom that allows learners to practice maneuvering a needle tip under ultrasound guidance. ${ }^{3}$ We also found that the same solid gel material used in that phantom can be used as an effective sound medium for scanning purposes. Ethics approval was obtained from the local Institutional Research Ethics Board to perform ultrasound scanning on the volunteer (one of the authors).

To construct this flexible gel medium, we obtained a heat-resistant glass container with the desired shape, and we poured melted gel (Gel Wax, Yaley Enterprises,

The first author is a recipient of the John Bradley Young Educator Award and the CAS Career Scientist Award in Anesthesia from the Canadian Anesthesiologists' Society.

B. C. H. Tsui, MD $(\bowtie) \cdot$ J. Tsui

University of Alberta Hospital, Edmonton, AB, Canada

e-mail: btsui@ualberta.ca
Redding, CA, USA) into the container to the desired thickness $(1-2 \mathrm{~cm})$. Once the melted gel had cooled down, it could be removed easily from the container as a flexible, transparent, ready-to-use pad. Using this gel pad (Figure), we were able to achieve an excellent image with superior probe stability and contact, which we attributed to the pad's flexibility. We found this property well-suited for sonoanatomy of the airway; the gel pad provided an effective medium for scanning the uneven surface of the neck, and we were able to localize the underlying anatomical structures without the use of additional liquid gel. As illustrated, the ultrasound images obtained with the gel pad and standard liquid gel were very consistent, with the only difference being that the gel pad provided a wider scanning area. This finding shows that the gel pad can be an effective substitute medium for educational purposes. In fact, the authors and a few of the authors' colleagues have used this gel pad on each other to gain experience and learn the sonoanatomy of the airway.

The gel pad described here may also have clinical applications during pre-scanning processes. When attempting to image structures under an uneven surface, the use of a gel pad as an ultrasound medium has the potential to provide ultrasound users with a high-quality image and a wider scanned region during the initial survey-scan. However, it would be quite impractical to perform realtime blocks with the pad as it would be necessary to insert the needle through the pad immediately after visualizing the target structure. Since the safety and sterility of this melted gel is unknown and cannot be ensured, it is important that this medium be used only for pre-scanning purposes and not for real-time guidance to avoid the possibility of inadvertent contamination of the patient from the gel pad. Thus, once the target has been located using the gel pad and the probe is in a satisfactory position, the pad 

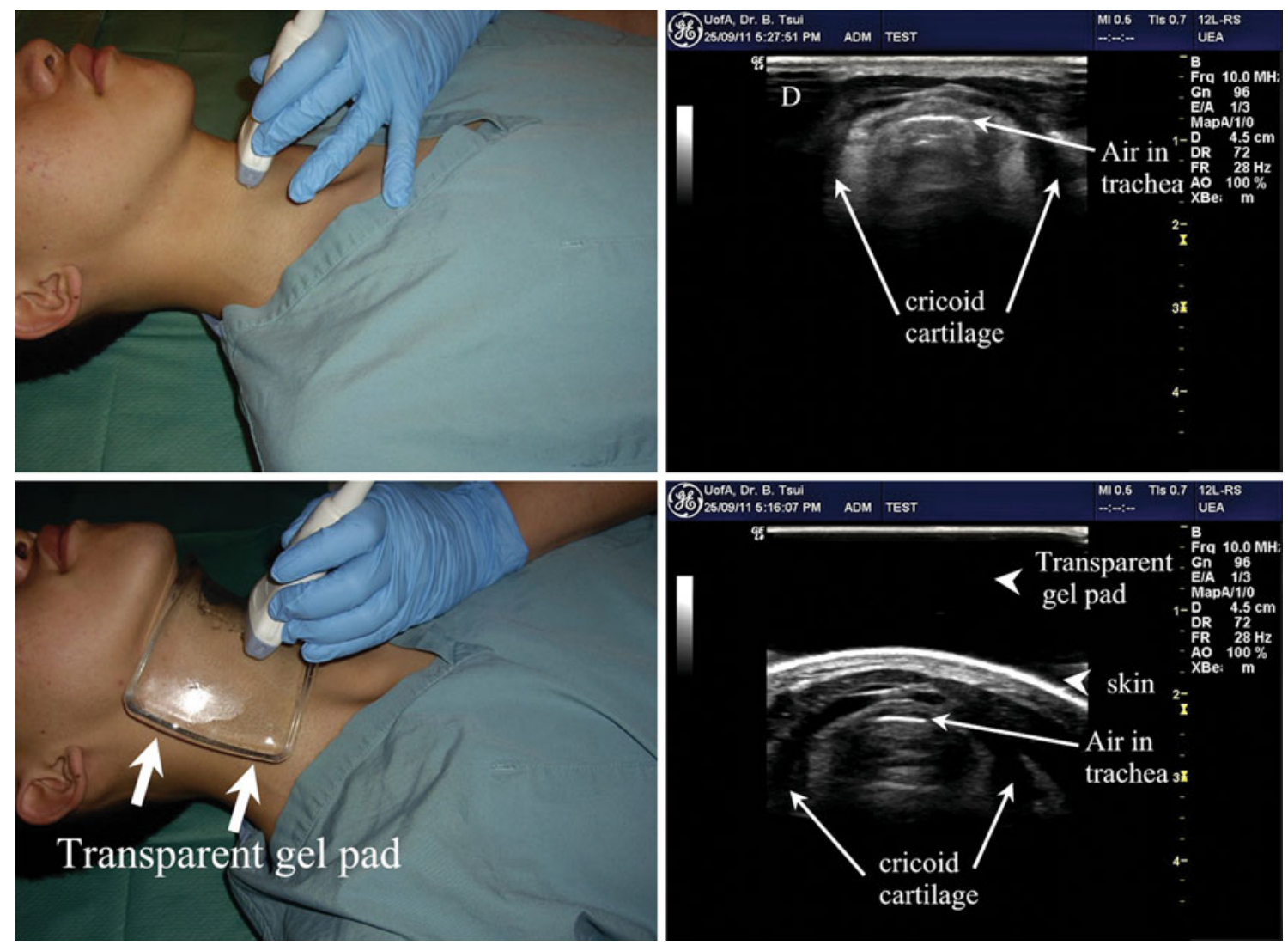

Figure Ultrasound images of airway with and without gel pad. (Top right) The image obtained without the gel pad is narrower and only part of cricoid cartilage is seen due to incomplete contact between the transducer and the skin with drop-out area (D). (Bottom right) The

image obtained with the gel pad allows full visualization of the target area using a compact ultrasound machine (12L-RS probe, Logic e, GE Medical Systems, WI, USA)

should be removed. The patient's skin that had been in contact with the gel pad should be thoroughly cleaned and sterilized in the standard fashion in preparation for ultrasonography using traditional sterile liquid gel.

Competing interests None declared.

2. Tsui BC. Dextrose $5 \%$ in water as an alternative medium to gel for performing ultrasound-guided peripheral nerve blocks. Reg Anesth Pain Med 2009; 34: 525-7.

3. Tsui BC, Tsui J. Reusable phantom with feedback signal for ultrasound needle tip control. Reg Anesth Pain Med 2012 (in press).

\section{References}

1. Dohgomori H, Shiba M, Okamoto K. Application of normal saline to ultrasonography as an alternative to gel for internal jugular venous cannulation. Can J Anesth 2007; 54: 246. 